LES ANNALES Les Annales de droit

DE DROIT

9 | 2015

Varia

\title{
Essai d'explication du déficit de garantie de la liberté politique au Togo
}

Explaining Why and How the Guarantee of Political Freedom in Togo has Failed

\section{Sasso Pagnou}

\section{(2) OpenEdition}

Journals

Édition électronique

URL : http://journals.openedition.org/add/363

DOI : $10.4000 /$ add.363

ISSN : 2606-1988

Éditeur

Presses universitaires de Rouen et du Havre

\section{Édition imprimée}

Date de publication : 1 juillet 2015

Pagination : 157-186

ISBN : 979-10-240-0516-4

ISSN : 1955-0855

\section{Référence électronique}

Sasso Pagnou, "Essai d'explication du déficit de garantie de la liberté politique au Togo », Les Annales de droit [En ligne], 9 | 2015, mis en ligne le 08 janvier 2018, consulté le 10 décembre 2020. URL : http:// journals.openedition.org/add/363; DOI : https://doi.org/10.4000/add.363 


\section{Essai d'explication du déficit de garantie de la liberté politique au Togo}

\section{Sasso PAGNOU}

Dans les démocraties libérales, l'existence de la Constitution est souvent liée à la garantie des libertés. Ce lien découle non seulement du fait qu'historiquement l'adoption de nouvelles constitutions correspond à la volonté des peuples de s'autodéterminer par rapport à une puissance étrangère ou à un groupe minoritaire les opprimant ${ }^{1}$, mais surtout de la définition même de la Constitution. Une définition de la liberté politique et de la constitution permet de relever la consubstantialité de ces deux notions. Selon Montesquieu, la liberté, dans une société où il y a des lois, est «le droit de faire tout ce que les lois permettent ${ }^{2}$ ». Cette définition se rapproche de la liberté civile de Jean-Jacques Rousseau, c'est-à-dire une liberté limitée par la volonté générale à travers la loi ${ }^{3}$. Ces deux définitions sous-entendent «la participation des hommes au choix de leur gouvernement, au processus de la législation, et au contrôle de l'administration ${ }^{4}$ ", exigence ${ }^{5}$ dont le respect atteste de plus en plus de la garantie de la liberté politique. Elles suggèrent une définition et une protection du domaine des droits et libertés par le droit, exercice auquel se livre la Constitution. En effet, dans sa conception matérielle, la Constitution est l'ensemble des règles juridiques les plus importantes ${ }^{6}$

1. Les premières constitutions des pays africains sont adoptées lors des indépendances marquant la fin du colonialisme se distinguant, comme le relève Aimé Césaire, par des «rapports de domination et de soumission ». Voir: Aimé Césaire, Discours sur le colonialisme, Paris-Dakar, Présence africaine, 2004, p. 23. La Constitution américaine de 1787 est intervenue après une guerre d'indépendance qui a duré de 1775 à 1783 . Il en de même de la Constitution française de 1792 suivant de près la Révolution française de 1789 dont l'une des causes était le rejet de l'absolutisme.

2. Montesquieu, De l'esprit des lois, livre XI, chap. 3.

3. Voir: Jean-Jacques Rousseau, Du contrat social, livre I, chap. 1.8.

4. Voir: Friedrich Hayek, La Constitution de la liberté, Raoul Audouin et Jacques Garello (trad.), Paris, Litec, 1994, p. 13.

5. C'est une exigence des instruments internationaux des droits de l'homme annexés au préambule de la Constitution. 
définissant le statut de l'État et celui des gouvernants ${ }^{7}$. Il faut, pour comprendre ce lien, relever que la notion de statut implique, en ellemême, une protection. En effet, qu'on le définisse comme l'ensemble des textes régissant le fonctionnement d'une société ou comme l'ensemble des dispositions fixant les garanties fondamentales d'une collectivité, le statut assure la paix sociale au sein de la société ou de la collectivité donnée et préserve leurs membres des éventuelles dérives des dirigeants. Dans ce sens, le respect de ce double statut garantit la liberté des citoyens à travers la limitation du pouvoir politique. L'affirmation de Fauré selon laquelle la Constitution est « une garantie des gouvernés, [...] une conquête de la liberté par ceux qui, jusqu'alors, ont été soumis à la force mécanique de la domination ${ }^{8}$ " tend aussi à relever ce lien. Les « révolutionnaires » français de 1789 , convaincus aussi de l'existence de ce lien, affirmeront sans ambages que " toute société dans laquelle la garantie des droits n'est pas assurée ni la séparation des pouvoirs déterminée, n'a point de Constitution ${ }^{9}$ ".

Ce lien se traduit par la présence de certains principes et mécanismes dans les constitutions libérales: l'égalité et la liberté des citoyens, la division du pouvoir politique, l'existence d'une autorité judiciaire et les conditions de dévolution du pouvoir politique.

Ces principes et mécanismes, prévus expressément pour soumettre le pouvoir politique au droit, se retrouvent aussi dans la Constitution togolaise du 14 octobre 1992. La Déclaration universelle des droits de l'homme et la Charte africaine des droits de l'homme et des peuples auxquelles renvoie le préambule de la Constitution affirment l'égalité des citoyens. La Constitution distingue, en outre, trois types de pouvoirs qu'elle confie à trois organes différents ${ }^{10}$.

La Constitution de 1992 est, de ce fait, porteuse de principes et valeurs tendant à garantir la liberté politique mais dont le respect peine à s'imposer comme l'atteste la récente résolution de la Fédération internationale des droits de l'homme (FIDH) relevant la persistance de la torture au

6. Voir: Jean Gicquel et Jean-Éric Gicquel, Droit constitutionnel et institutions politiques, Paris, Montchrestien, 2007, p. 171.

7. Georges Burdeau, Traité de science politique, II : L'État, Paris, LGDJ, 1980, p. 169.

8. Yves-André Fauré, «Les constitutions et l'exercice du pouvoir en Afrique. Pour une lecture différente des textes ", Politique africaine, $\mathrm{n}^{\circ} 1,1981$, p. 35 .

9. Article 16 de la Déclaration des droits de l'homme et du citoyen de 1789 .

10. Voir les titres III, IV et VIII. 
Togo ${ }^{11}$. La balance des pouvoirs reste encore fictive dans la mesure où l'Assemblée nationale passe encore, pour des raisons qui seront ultérieurement présentées, plus pour une chambre d'enregistrement qu'une chambre parlementaire. Quant à l'indépendance de la justice, elle est encore attendue ${ }^{12}$.

On constate donc un déficit de garantie de la liberté politique malgré l'existence de principes constitutionnels ayant un tel objet. Les mêmes principes ayant atteint les résultats escomptés dans d'autres pays ${ }^{13}$, les raisons de ce déficit ne peuvent qu'être recherchées dans leurs conditions d'application. Ceci suppose donc, en plus du formalisme permettant l'appréhension des exigences du corpus juridique togolais s'agissant de la garantie de la liberté politique, l'adoption d'un réalisme juridique exigeant de "partir d'une description empirique des faits juridiques observables (le droit existant réellement ${ }^{14}$ )».

Le choix des deux théories pour expliquer le déficit de garantie de la liberté politique conduit à une présentation et à une explication des principes et mécanismes constitutionnels conçus pour garantir la liberté politique, d'une part, et leur application, d'autre part. L'objectif est de rechercher à la fois la part des insuffisances propres (internes) au dispositif juridique et rendant difficile la garantie de la liberté politique et celle des acteurs et de la société. La notion d'acteurs désigne, ici, les politiques, c'est-à-dire ceux dont dépendent, en premier lieu, l'application et la protection de la Constitution. On constate à leur niveau une volonté de contourner la Constitution par des artifices juridiques. La

11. Voir: FIDH, Note sur la situation des droits de l'homme au Togo (Les engagements des autorités doivent se transformer en actes concrets), présentée à l'occasion de l'examen du rapport du Togo par la Commission africaine des droits de l'homme et des peuples, $50^{\mathrm{e}}$ session, Banjul, 24 octobre7 novembre 2011. La note est disponible en ligne: http://www.fidh.org/IMG/ pdf/togonote2011.pdf (consulté le 24 novembre 2014). La même organisation a récemment condamné la pratique persistance de la torture et toute autre forme de traitements cruels, inhumains ou dégradants au Togo. Voir: FIDH, Résolution d'urgence sur la situation des droits humains au Togo, $38^{\mathrm{e}}$ congrès de la FIDH, Istanbul, 23-27 mai 2013, http://www.fidh.org/fr/afrique/Togo,81/ resolution-d-urgence-sur-le-togo-proposition-d-ammandement-13355 (consulté le 24 novembre 2014).

12. La résolution de la FIDH précédemment évoquée invite les autorités togolaises à respecter l'indépendance de la justice, le droit à un procès équitable, et la présomption d'innocence des personnes accusées.

13. Outre les grandes démocraties libérales occidentales, on peut citer l'exemple des pays voisins comme le Ghana et le Bénin.

14. Voir: Éric Millard, "Le réalisme », dans Denis Alland et Stéphane Rials (dir.), Dictionnaire de la culture juridique, Paris, PUF, 2003, p. 1297. 
société désigne, elle, l'environnement socio-économique et culturel. Les déséquilibres de cet environnement empêchent l'équilibre des pouvoirs et vident les modalités d'accession au pouvoir de leur sens. Dans un cas comme dans l'autre, on constate un impact négatif des facteurs culturels et économiques sur la garantie des droits.

Cet impact négatif se résume en deux processus d'émasculation ${ }^{15} \mathrm{de}$ la Constitution : le déclassement de la Constitution et l'anéantissement des principes de limitation du pouvoir politique.

\section{Le déclassement implicite de la Constitution}

Dans son acceptation première, le déclassement est une opération consistant à modifier, par un acte réglementaire, une disposition de forme législative ne devant pas, en principe, relever du domaine de la loi. Par analogie, le déclassement de la Constitution désigne, ici, un certain nombre d'actes tendant à la faire passer de sa situation de norme fondamentale ${ }^{16}$ à une loi comme les autres. Ce processus repose sur une remise en cause de la suprématie de la Constitution.

\subsection{L'affirmation de la suprématie de la Constitution par le peuple}

La « déconstitutionnalisation » de la constitution, constatée par Aïvo, dans le cadre de la crise de la normativité de la Constitution en Afrique ${ }^{17}$, soulève inévitablement la question de la normativité de la Constitution dont l'affirmation exige que soient présentées les dispositions constitutionnelles desquelles elle peut être déduite. La conviction de cette normativité soulève à son tour la question de l'État de droit dont le respect se présente également comme une exigence constitutionnelle.

15. Cette émasculation de la Constitution est la conséquence de l'émasculation de la démocratie dans les pays africains que relève Holo; voir: Théodore Holo, "Démocratie revitalisée ou démocratie émasculée? Les constitutions du renouveau démocratiques dans les États de l'espace francophone: régimes juridiques et systèmes politiques ", Revue béninoise des sciences juridiques et administratives, $\mathrm{n}^{\mathrm{o}} 16,2006$, p. 17-41.

16. Voir le dernier alinéa du préambule de la Constitution.

17. Voir: Frédéric Joël Aïvo, "La crise de la Constitution en Afrique ", RDP 2012, p. $147-148$. 


\subsubsection{La reconnaissance du caractère de loi fondamentale}

Aux termes du dernier alinéa du préambule de la Constitution togolaise du 14 octobre 1992, le peuple togolais approuve la dite Constitution comme loi fondamentale de l'État.

La notion de loi fondamentale renvoie à la normativité postulée par Hans Kelsen dans sa théorie du droit. On peut retenir de la théorie kelsénienne que c'est sa conformité à une norme supérieure qui confère à une norme inférieure sa validité juridique. La validité se présente, de ce fait, comme "le mode d'existence spécifique des normes». Découle de cette affirmation la question de la validité juridique de la Constitution, elle-même, placée au sommet de la pyramide. Selon Kelsen, il existerait à l'échelon supérieur de la Constitution, une norme supérieure non posée, ne faisant pas partie du droit positif et répondant à « une hypothèse logique transcendantale ${ }^{18}$ ».

La déduction de la validité juridique de la Constitution d'une norme supérieure non posée, donc "supposée ${ }^{19}$ ", relève de l'irréalisme comme le fait, d'ailleurs, remarquer Paul Amselek, en ce que la norme supérieure supposée serait, à la fois « un acte de connaissance qui aurait des effets prescriptifs » et "une norme de conduite supposée en vigueur par enchantement, sans avoir fait l'objet d'un acte de commandement ${ }^{20}$ ». L'hypothèse d'une norme supposée s'imposant à tous est donc difficile à admettre dans le cadre de l'État. La justification de la normativité juridique de la Constitution togolaise du 14 octobre 1992 par l'existence d'une norme supérieure non posée est contraire à la logique positiviste revendiquée par Kelsen. Il paraît alors nécessaire de recourir à une autre théorie. On choisira, dans ce sens, la théorie contractuelle de la Constitution et plus précisément celle d'un pacte d'association.

L'explication de la normativité juridique de la Constitution par la théorie contractuelle remonte à Thomas Hobbes. Dans le Léviathan (1651), l'auteur décrit le pacte d'association comme le contrat par lequel les hommes décident librement de s'unir pour conférer à une seule personne ou à une assemblée la mission d'agir à leurs places dans un certains nombres de domaines. L'idée sera approfondie par d'autres

18. Hans Kelsen, Théorie pure du droit, Paris, LGDJ, 1999, p 13 et 266.

19. Michel Troper, "Normativisme ", dans Denis Alland et Stéphane Rials (dir.), Dictionnaire de la culture juridique, op. cit., p 1074 et suiv.

20. Paul Amselek, "Une fausse idée claire: la hiérarchie des normes juridiques ", dans Renouveau du droit constitutionnel. Mélanges en l'honneur de Louis Favoreu, Paris, Dalloz, 2007, p. 990. 
auteurs notamment Pufendorf, Rousseau et Vattel, qui s'intéressent plus à la notion d'association.

Pour Pufendorf, tout comme pour Hobbes, tout commence par le pacte d'association qui «donne son existence au corps politique ${ }^{21}$ ». Rousseau voit, lui, dans ce pacte d'association, l'acte qui défend et protège de toute la force commune la personne et les biens de chaque associé 22 .

L'association de ces différentes idées constitue, en quelque sorte, la matrice des éléments du statut de l'État qu'est la Constitution. L'idée est bien résumée par Vattel: "Cette constitution n'est dans le fonds autre chose que l'établissement dans l'ordre dans lequel une nation se propose de travailler en commun à obtenir les avantages en vue desquels la société politique s'est établie ${ }^{23}$.»

La nature contractuelle de la Constitution est aussi affirmée en droit positif. La Constitution du Massachussets de 1780 se définit, en ce sens, comme « un contrat social, par lequel tout le peuple contracte avec chaque citoyen, et chaque citoyen avec tout le peuple».

La nature contractuelle des Constitutions étant reconnue et les contrats étant des normes ${ }^{24}$, il est donc possible d'affirmer la normativité juridique de la Constitution togolaise du 14 octobre 1992. Cette normativité reste, toutefois, conditionnée par la possibilité de sanctionner les atteintes à la Constitution quand on sait que l'existence de la norme est souvent associée à celle d'une sanction ${ }^{25}$. Or, il n'est pas possible, sur ce point, malgré l'existence d'une Cour constitutionnelle, d'entrevoir des sanctions aux atteintes à la Constitution en référence à la «théorie de l'aiguilleur », développée par le doyen Favoreu ${ }^{26}$, et selon laquelle le juge constitutionnel se présente moins comme un censeur qu'un aiguilleur se bornant à indiquer au législateur la voie à suivre. Cette affirmation n'est, en réalité, vérifiée que sur le plan interne car, sur le plan

21. Carlos-Miguel Pimentel, «Du contrat social à la norme suprême: l'invention du pouvoir constituant», Jus politicum, $\mathrm{n}^{\circ}$ 3, 2009, p. 12.

22. Jean-Jacques Rousseau, Du contrat social, livre I, chapitre vi.

23. Emer de Vattel, Le Droit des gens ou principes de la loi naturelle appliqués à la conduite et aux affaires des nations et des souverains, Paris, Janet et Cotelle, 1820, t. I, p. 68-69.

24. Michel Troper, «Normativisme», dans Denis Alland et Stéphane Rials (dir.), op. cit., p. 1077.

25. Hans Kelsen, Théorie pure du droit, op. cit., p. 71 ; du même auteur, Théorie générale du droit et de l'État, Béatrice Laroche et Valérie Faure (trad.), Paris, LGDJ, 1997, p. 177.

26. Louis Favoreu, «Les décisions du Conseil constitutionnel dans l'affaire des nationalisations ", $R D P$ 1982, p. 419. 
international, des organisations régionales telles l'Union économique et monétaire ouest africaine (UEMOA) et la Communauté des États de l'Afrique de l'Ouest (CEDEAO) prévoient des sanctions (quelquefois ciblées) en cas d'atteinte à l'ordre constitutionnel. Mais l'argument de taille sur cette question est l'absence de lien nécessaire entre l'existence d'une norme juridique et la sanction. Kelsen admet lui-même l'existence d'une catégorie de normes dépourvues de sanction. Il cite en exemple les "obligations naturelles", qu'il définit comme "l'obligation à une prestation dont l'exécution ne peut pas être réclamée par une action en justice et dont la non-exécution ne permet pas l'exécution civile ». Cette absence de lien nécessaire entre la sanction et l'existence d'une norme est reconnue par d'autres auteurs notamment Herbert Hart, qui distingue en droit entre, d'une part, les règles primaires prescrivant une action ou une omission et, d'autre part, les règles secondaires prévoyant des procédures d'introduction des règles primaires. L'existence de cette seconde catégorie de règles, se présentant essentiellement comme des lois d'habilitation, n'est pas conditionnée par l'existence d'une quelconque sanction. On retrouve dans tous les systèmes juridiques des règles non prescriptives et de ce fait non assorties de sanctions. On peut citer, dans ce sens, l'exemple de règles accordant des pouvoirs aux autorités publiques ${ }^{27}$. La fragilité de ce dernier argument doit, donc, conduire à admettre définitivement la normativité de la Constitution du 14 octobre 1992 et son corollaire d'État de droit.

La Constitution togolaise du 14 octobre 1992 dispose donc d'une normativité juridique en vertu de laquelle elle prescrit le respect de l'État de droit.

\subsubsection{L'exigence de respect de la Constitution}

L'article 1 de la Constitution togolaise du 14 octobre 1992 fait de la République togolaise un État de droit.

Tel que conceptualisé par les auteurs allemands, l'État de droit (Rechtsstaat) " protège et encourage le développement de toutes les forces naturelles, développement reconnu par le peuple comme objectif de vie de l'individu et de l'ensemble de la société ${ }^{28}$ ». En référence à ces auteurs, Carré de Malberg définit de façon beaucoup plus précise l'État

27. Alain Laquièze, "Sanction", dans Denis Alland et Stéphane Rials (dir.), op. cit., p. 1380.

28. Voir: Robert von Mohl, Encyclopädie der Staatswissenschaft, $2^{\mathrm{e}}$ éd., Fribourg et Tübingen, 1872, p. 106. 
de droit comme "un État qui, dans ses rapports avec ses sujets et pour la garantie de leur statut individuel, se soumet lui-même à un régime de droit, et cela en tant qu'il enchaîne son action sur eux par des règles dont les unes déterminent les droits réservés aux citoyens, dont les autres fixent par avance les voies et moyens qui pourront être employés en vue de réaliser les buts étatiques ${ }^{29} \ldots »$

La soumission de l'État à un "régime de droit» se présente, donc, comme le trait fondamental de l'État de droit. Sont soumis à ce régime, non seulement "les autorités administratives" mais aussi le "corps législatif ${ }^{30}$ ». La soumission au droit des autorités administratives et du corps législatif, organes chargés de l'exercice du pouvoir politique au sein de l'État, sous-entend la limitation du pouvoir politique par le droit.

La soumission du politique au droit va, donc, au-delà du simple respect de la légalité, exigence du « régime de l'État légal». Celui-ci tend à encadrer uniquement l'action administrative alors que l'État de droit limite à la fois l'autorité administrative et le corps législatif ${ }^{31}$. Hayek écrit dans ce sens: «L'État de droit, bien entendu, suppose que la légalité soit complète, mais cela ne suffit pas: si une loi donnait au gouvernement le pouvoir illimité d'agir comme il l'entend, toutes ses actions seraient légales, mais il n'y aurait incontestablement pas d'État de droit. L'État de droit implique donc davantage que le constitutionnalisme: il requiert que toutes les lois se conforment à certains principes ${ }^{32}$. "Dans sa décision du 16 janvier 1996, le Conseil constitutionnel français relève aussi l'obligation pour le législateur de se soumettre à certains principes. Il juge, en effet, que rien "ne saurait dispenser le législateur, dans l'exercice de sa compétence, du respect des principes et des règles de valeur constitutionnelle qui s'imposent à tous les organes de l'État ${ }^{33} »$. Le mot «principe » utilisé par Hayek et le Conseil constitutionnel suggère la soumission du législateur, non pas seulement, au texte de la Constitution mais au bloc de constitutionnalité et à l'esprit de la Constitution ${ }^{34}$.

29. Raymond Carré de Malberg, Contribution à la théorie générale de l'État [1920-1922], Paris, Dalloz, 2004, t. I, p. 489.

30. Ibid., p. 492.

31. Comme le relève le doyen Favoreu, l'ensemble de la doctrine s'accorde sur le fait que l'État de droit suppose que toute autorité, y compris le législateur, soit soumise à la règle de droit. Voir: Louis Favoreu, «Les décisions du Conseil constitutionnel dans l'affaire des nationalisations", art. cité, p. 301.

32. Friedrich Hayek, La Constitution de la liberté, op. cit., p. 205.

33. Voir Cons. const., déc. 16 janvier 1982, $\mathrm{n}^{\circ}$ 81-132 DC sur la loi de nationalisation, consid. 18.

34. Louis Favoreu, op. cit., p. 401. 
La limitation du corps législatif agissant en qualité de législateur ordinaire est unanimement admise, car expressément prévue par l'article 84 de la Constitution définissant le domaine de la loi et par l'article 104 relatif au contrôle de constitutionnalité des lois ${ }^{35}$. Par contre, la limitation de ce corps agissant en qualité de pouvoir constituant dérivé l'est moins, la souveraineté du pouvoir constituant étant affirmée par la jurisprudence ${ }^{36}$ et par une partie de la doctrine ${ }^{37}$.

Bien que soit reconnue la souveraineté du législateur agissant en sa qualité de pouvoir constituant qu'il soit dérivé ou originaire, il importe de s'interroger sur l'étendue du pouvoir de révision de la Constitution dont il dispose.

Se fondant sur la notion de supra-constitutionnalité, dont il est l'auteur, Carl Schmitt estime que «les décisions fondamentales de la constitution relèvent du pouvoir constituant du peuple [...] et non de la compétence des instances habilitées aux modifications et révisions légiconstitutionnelles ${ }^{38}$ ». L'affirmation de Schmitt laisse, donc, entrevoir une limitation du législateur agissant en sa qualité de pouvoir constituant dérivé. Cette opinion est contestée par une partie de la doctrine notamment par Léon Duguit et Georges Vedel. Dans ce sens, Léon Duguit affirme à propos de la loi constitutionnelle française de 1884 interdisant de réviser la forme républicaine du gouvernement que "cette disposition a été votée par une assemblée nationale de révision et [...] elle peut être modifiée ou abrogée par une autre assemblée de révision [...], par conséquent, tant que ce texte existe l'assemblée nationale ne peut pas changer la forme de gouvernement, elle n'a qu'à l'abroger, et la chose est faite, elle pourra très constitutionnellement changer la forme républicaine du gouvernement ${ }^{39}$ ».

La finalité majeure de la Constitution étant la protection des droits des citoyens, il est possible d'affirmer l'interdiction pour le législateur, agissant en sa qualité de pouvoir constituant dérivé, de modifier, non pas la lettre de certaines dispositions, notamment celles relatives aux

35. On peut, certes, relever la critique du professeur Lacharrière qui n'a pas fait florès. L'auteur conteste le contrôle de constitutionalité des lois au nom de la souveraineté. Voir: René de Lacharrière, «Opinion dissidente », Pouvoirs, $\mathrm{n}^{\circ}$ 13, 1980, p 17.

36. Voir: Cons. const., déc. 6 novembre 1962, $\mathrm{n}^{\circ}$ 62-20 DC sur la loi référendaire à l'élection et $\mathrm{n}^{\mathrm{0}}$ 92-312 DC.

37. Voir: Christian Bigaut, «La Constitution demeure la norme suprême : le pouvoir constituant est souverain ", Rev. adm. 2003, p. 605-608.

38. Carl Schmitt, La Théorie de la Constitution, Paris, PUF, 1989, p. 241.

39. Léon Duguit, Traité de droit constitutionnel, Paris, Ancienne librairie Fontemoing, 1924 , t. IV, p. 540. 
droits et libertés des citoyens, mais l'esprit de ces dispositions. C'est cet esprit qui confère à la Constitution son identité et sa continuité lorsqu'il est respecté, comme l'explique Carl Schmitt qui l'assimile à la supra-constitutionnalité ${ }^{40}$. La supra-constitutionnalité recouvre, de ce fait, la séparation des pouvoirs et le respect des droits de l'homme. C'est dans ce sens qu'il faut comprendre l'article 16 de la Déclaration des droits de l'homme et du citoyens aux termes duquel «toute société dans laquelle la garantie des droits n'est pas assurée ni la séparation des pouvoirs déterminée n'a point de Constitution ». En clair, le corps législatif, agissant en qualité de pouvoir constituant dérivé, ne peut pas apporter à la Constitution des modifications violant le principe de la séparation des pouvoirs et les droits de l'homme. C'est ce qu'exprime le paragraphe 6 de l'article 144 de la Constitution interdisant la révision de la forme républicaine. Il faut, en effet, comprendre par république non pas un régime mais un certain nombre de principes et valeurs fondant la république.

\subsection{La remise en cause de la suprématie de la Constitution par le politique}

La remise en cause de la suprématie de la Constitution correspond, avant tout, au rejet de son caractère «sacré» interdisant sa transgression. Ce rejet commence par la banalisation de la Constitution pour aboutir sur le non-respect de l'exigence de l'État de droit.

\subsubsection{La banalisation de la Constitution}

La banalisation de la Constitution est le déni de son caractère fondamental donnant la possibilité de sa limitation. Le génie politique a développé, dans ce sens, au moins trois types de stratagèmes: les révisions constitutionnelles à l'objet peu licite et controversé ${ }^{41}$, les interprétations de la Constitution trahissant son esprit et l'abstention de voter les lois organiques devant compléter la Constitution.

Il faut, à titre illustratif, citer l'interprétation et l'application par le chef de l'État, en 1992, de l'article 66 de la Constitution. Dans sa version originelle de 1992, cet article disposait : «Le président de la République nomme le Premier ministre dans la majorité parlementaire...»

L'interprétation de cet article par le chef de l'État comme lui donnant un pouvoir discrétionnaire de nomination du Premier ministre dans la

40. Carl Schmitt, op. cit.

41. Frédéric Joël Aïvo, «La crise de la Constitution en Afrique», art. cité, p. 154. 
majorité parlementaire sans considération du choix de cette majorité, afin, certainement, d'attiser les rivalités entre ses adversaires de la majorité, a laissé un précédent sur la possibilité de jouer avec le texte fondamental. Cet essai fut confirmé par le différend opposant le président de la République au Premier ministre en l'absence (certainement voulue) de la loi organique prévue par le dernier paragraphe de l'article 70 pour déterminer les autres emplois auxquels il est pourvu en Conseil des ministres. La banalisation s'accentue à partir de 1998, période durant laquelle la Constitution est livrée à la vindicte populaire : la Constitution, loi fondamentale, est dénoncée et quotidiennement dénigrée par des manifestants demandant sa révision.

Deux révisions interviendront. Si, d'une façon générale, les révisions constitutionnelles sont normales et répondent à une nécessité d'adaptation des textes à l'évolution de la société ${ }^{42}$, celles intervenues au Togo participent à la banalisation de la Constitution par leurs ampleurs, leurs contenus et les conditions dans lesquelles elles sont intervenues. La révision du 31 décembre 2002 a porté sur le préambule et 48 des 159 articles de la Constitution, soit environ le tiers de la Constitution. Cette révision constitue, comme le relève Abdou-Salami, une revanche sur la Conférence nationale ${ }^{43}$ et surtout la remise en cause de la limitation des mandats. Au Togo, comme dans la plupart des pays africains, cette limitation a été voulue par les Conférences nationales pour éviter les présidences à vie, les coups d'État et l'instabilité en découlant ${ }^{44}$. Au-delà de cette remise en cause de ce qui se présente comme l'un des objectifs fondamentaux de la Constitution, le fait de se servir de la Constitution comme objet de revanche ou même de vengeance relève du dévoiement de la révision et, donc, de la banalisation de la Constitution. Le mot «toilettage » utilisé pour qualifier cette révision, en contradiction avec son ampleur, participe à cette logique.

La banalisation de la Constitution atteint son paroxysme avec la révision constitutionnelle du 6 février 2005 portant sur les articles 65

42. Comme le relève Lavroff: «La Constitution ne peut être stable quand la société évolue sauf à courir le risque de la crise politique majeure aboutissant à un changement de régime. "Voir: Dimitri Georges Lavroff, "La Constitution et le temps ", dans Mélanges Philippe Ardant. Droit et politique à la croisée des cultures, Paris, LGDJ, 1999, p. 212.

43. Voir: Mama-Sani Abdou-Salami, "La révision constitutionnelle du 31 décembre 2002: une revanche sur la Conférence nationale de 1991 ", Revue béninoise de sciences juridiques et administratives, $\mathrm{n}^{\circ} 19,2007, \mathrm{p}$. 53-94.

44. Elles constituent, comme le relève Aïvo l'une des «garanties de sureté démocratique des régimes démocratiques africains» (art. cité, p. 156). 
et 144 de la Constitution. Cet acte soulève la question du respect des interdictions de révision de la Constitution. De façon générale, ces interdictions peuvent porter, d'une part, sur des idées ou valeurs de la Constitution et renvoient, de ce fait, à la supra-constitutionnalité déjà évoquée ou, d'autre part, sur les circonstances dans lesquelles la Constitution ne peut être changée. La révision du 6 février se range dans la seconde série des interdictions puisque violant le paragraphe 5 de l'article 144 de la Constitution interdisant toute révision de la Constitution en période d'intérim ou de vacance du pouvoir. La portée de ces interdictions est discutée en doctrine. Pour certains auteurs, notamment Léon Duguit ${ }^{45}$ et Georges Vedel, il suffit de modifier le texte édictant l'interdiction et la Constitution pourra être modifiée. C'est la voie choisie par l'Assemblée nationale togolaise qui, pour réviser l'article 65, révisa simultanément l'article 144. Les deux auteurs se fondent sur la souveraineté. Le même argument est avancé en droit positif et par les politiques ${ }^{46}$.

La banalisation de la Constitution ne vient pas, dans ce sens, du non-respect de l'interdiction de révision. L'Assemblée nationale peut, comme l'ont suggéré Duguit et Vedel, préalablement lever l'interdiction et ensuite réviser les dispositions voulues. Le véritable problème est le non-respect par cette assemblée des délais et des procédures constitutionnelles. Les articles 65 et 144 sont révisés simultanément. L'abrogation de l'article 144 interdisant de réviser en cas de vacance de pouvoir n'a pas été promulguée et donc n'était pas en vigueur avant que n'intervienne la révision de l'article 65. Cette précipitation suggère une certaine fraude à la Constitution ou son instrumentalisation ${ }^{47}$.

45. Léon Duguit, Traité de droit constitutionnel, op. cit., p. 540.

46. Le président Jefferson écrit dans ce sens : «Chaque génération est indépendante de celle à laquelle elle succède, comme celle-là même l'était de la génération qui l'a précédée. Elle a, comme l'une et l'autre, le droit de choisir la forme de gouvernement qu'elle juge le plus favorable à son bonheur, et par conséquent, d'accommoder aux circonstances dans lesquelles elle se trouve placée, les institutions qu'elle a reçues de ses pères. » Voir : «Lettre à Samuel Kerchiel du 12 juillet 1816 », dans Mélanges politiques et littéraires, extraits de mémoires et de la correspondance de Thomas Jefferson, Paris, Paulin, 1883, t. II, p. 287. L'article 28 de la Déclaration des droits de l'homme et du citoyen du 24 juin 1793 : «Un peuple a toujours le droit de revoir, de reformer et réviser une Constitution. Une génération ne peut assujettir à ses lois les générations futures. »

47. Sur la notion de fraude à la Constitution, voir: Georges Liet-Veaux, «La fraude à la constitution: essai d'une analyse juridique des révolutions communautaires récentes », $R D P 1943$, p. 116-150. S'agissant spécialement de la fraude à la constitution dans les pays africains, voir: Adama Kpodar, «Bilan sur un demi-siècle de constitutionnalisme en Afrique noire francophone", Revue électronique Afrilex, 


\subsubsection{La relativisation de l'État de droit}

L'effectivité de l'État de droit dépend de la mise en œuvre de deux principes substantiels de la Constitution : l'ordre et la liberté ${ }^{48}$.

Si l'ordre semble assuré par la légalité, la liberté est présumée lorsque sont proclamées et respectées l'égalité entre les citoyens et l'indépendance de la justice.

La légalité peut être comprise de deux façons. Stricto sensu, elle renvoie à la conformité des actes des autorités administratives à la loi. Lato sensu, elle exige le respect de l'ordre juridique tout entier, notamment le respect de la hiérarchie des normes. Cette seconde acception est adoptée dans le cadre de la présente analyse.

Le respect de la légalité est une exigence des paragraphes 1 et 3 de l'article 104 de la Constitution confiant le contrôle de constitutionnalité des lois à la Cour constitutionnelle, d'une part, et de l'article 119 posant les principes d'unité juridictionnelle et de séparation des contentieux entre des juridictions administratives et des juridictions judiciaires.

Cette exigence constitutionnelle n'est respectée que de manière très relative. La Cour constitutionnelle est certes fonctionnelle depuis plusieurs années. Mais le contrôle de constitutionnalité des lois et la garantie des droits fondamentaux n'occupent qu'une place très relative dans sa jurisprudence largement dominée par le contentieux électoral ${ }^{49}$. Cela s'explique par les conditions de saisine de la Cour constitutionnelle réservée aux pouvoirs institués ${ }^{50}$ souvent à l'origine de la violation des droits fondamentaux garantis par la Constitution. Il y a lieu, malgré la possibilité offerte par le paragraphe 6 de l'article 104 de la Constitution à toute personne physique ou morale de soulever une exception d'inconstitutionnalité d'une loi au cours d'une instance judiciaire, d'ouvrir un débat sur les conditions de saisine de la Cour constitutionnelle surtout en ce qui concerne la violation des droit fondamentaux. La récente décision de la chambre judiciaire de la Cour suprême ${ }^{51}$ de joindre au fond l'exception d'inconstitutionnalité de l'article $447 \mathrm{du}$ Code de procédure pénale soulevée par les avocats des accusés d'atteinte

http://afrilex.u-bordeaux4.fr/bilan-sur-un-demi-siecle-de.html (consulté le 24 novembre 2014).

48. Sur ces principes, voir Simone Goyard-Fabre, Les Principes philosophiques du droit moderne, Paris, PUF, 1997, p. 251.

49. Cette jurisprudence est consultable en ligne: http://www.courconstitutionnelle.tg.

50. $\$ 104$ de l'article de la Constitution.

51. Cour suprême, chambre judiciaire, arrêt $\mathrm{n}^{\mathrm{o}} 105 \mathrm{du} 4$ août 2011, Ministère public et État togolais contre Kpatcha Gnassingbé et 32 autres. 
à la sûreté de l'État rend davantage nécessaire ce débat. Cette décision peut créer une jurisprudence permettant au juge ordinaire de ne pas surseoir à statuer et de saisir la Cour constitutionnelle en cas d'exception d'inconstitutionnalité soulevée par une personne. Une telle éventualité peut vider le paragraphe 6 de l'article 104 de la Constitution de tout son sens ou, au contraire, entraîner, dans le temps et avec une éventuelle modification de ce paragraphe, un contrôle de constitutionnalité diffus. La seconde possibilité permettrait une plus grande protection des droits et libertés des citoyens et donc une plus grande garantie de la liberté politique.

Le faible fonctionnement, des juridictions administratives va jusqu'à mettre en péril le respect de l'exigence de légalité lorsque l'on sait que le juge administratif garantit le respect de la loi, la continuité de la Constitution et protège les droits des administrés ${ }^{52}$. Les raisons de cette léthargie de la justice administrative sont de deux ordres comme l'expliquait Massina ${ }^{53}$, le poids de l'environnement sociologique et les freins d'ordre institutionnel dont l'importance appelle d'amples développements. L'auteur cite parmi les freins d'ordre institutionnel «la résistance passive de l'Exécutif » au fonctionnement des juridictions administratives et le refus du législateur de faire de la justice administrative une justice gratuite comme l'est la justice civile. La persistance de cette somnolence de la justice administrative, malgré l'exécution d'un programme national de modernisation de la justice, s'explique certainement par la peur de l'autorité de se voir contestée. S'agissant du second frein, d'ordre institutionnel, il est plus qu'évident que le caractère onéreux de la justice administrative en exclut une bonne partie des citoyens dans un pays où $52,7 \%$ de la population vit avec moins de deux dollars par jour $^{54}$. Il faut, en effet, noter que la somme de 10 ooo francs CFA exigée au titre de la consignation pour excès de pouvoir constitue une part non négligeable du revenu mensuel de bien des travailleurs ${ }^{55}$ ne pouvant, de ce fait, la consacrer à une procédure contentieuse à l'issue incertaine.

52. Jean-Marie Breton, «Légalité et État de droit: statut et perception du juge de l'administration (contribution à une réflexion transversale)», Revue électronique Afrilex, $\mathrm{n}^{\circ} 3,2003$, p. 72.

53. Palouki Massina, «Plaidoyer pour le fonctionnement de la juridiction administrative au Togo », Revue burkinabè de droit, $\mathrm{n}^{\text {os }}$ 19-20, 1991, p. 7-25.

54. Données disponibles en ligne: http://donnees.banquemondiale.org/indicateur/SI. POV.2DAY (consulté le 24 novembre 2014).

55. Si on fixe approximativement la valeur de 1 dollar à 500 francs CFA, les 10000 francs CFA feront 20 dollars qui peuvent permettre à 52,7 \% de la population de vivre pendant plus du tiers du mois. 
L'impossibilité pour certains de saisir la justice soulève la question de l'égalité entre les citoyens, deuxième condition de l'effectivité de l'État de droit.

L'égalité de tous les citoyens devant la loi sans distinction d'origine, de race, de sexe, de condition sociale ou de religion est affirmée par l'article 2 de la Constitution. La réalité de cette égalité témoigne de l'avancement de la démocratie et du respect des droits de l'homme proclamés par la Constitution dans la mesure où des liens de consubstantialité existent entre le principe d'égalité et les idées de démocratie et des droits de l'homme ${ }^{56}$. L'incapacité de la puissance publique à se déployer sur tout le territoire national rend difficile l'atteinte de cet objectif. Comment entrevoir, en effet, l'égalité dans un pays où des pans entiers de populations n'ont pas d'état civil et où certaines parties du territoire ne connaissent aucun service public?

Ces lacunes touchent aussi le service public de la justice, ce qui conduit à s'interroger sur son indépendance. Malgré l'existence d'un statut spécial des magistrats destiné à offrir aux juges les moyens de leur indépendance, la récurrence de ce sujet dans l'actualité scientifique et politique conduit encore aujourd'hui à envisager l'indépendance du pouvoir judiciaire comme un objectif à atteindre. La non-application du statut des magistrats voté déjà depuis plus d'une décennie témoigne des efforts à faire dans ce sens. L'une des conséquences de cette nonapplication du statut des magistrats est la privation des avantages financiers prévus par ce statut pour mettre le juge à l'abri de toute proposition pouvant altérer son indépendance de jugement. Cette situation serait l'une des causes de la corruption dans le monde judiciaire dénoncées depuis quelques années déjà ${ }^{57}$. La corruption de la justice rompt le principe d'égalité entre les citoyens, viole leurs droits, notamment celui de propriété, un élément à l'origine de la civilisation moderne ${ }^{58}$, et

56. Michel Borgetto, «L'article $1^{\text {er }}$ de la Constitution », dans Gérard Conac, François Luchaire et Xavier Prétot (dir.), La Constitution de la République française: analyses et commentaires, Paris, Economica, 2009, p. 153.

57. Allocution du Premier ministre lors de l'atelier du 9 juin 2008 portant sur la "Convention des Nations unies contre la corruption». Voir également HautCommissariat des Nations unies, Rapport sur le respect et la mise en ouvre des droits de l'homme et des libertés fondamentales dans l'administration de la justice au Togo, décembre 2013 ( 55 p). Le rapport relève des pratiques de corruption chez les magistrats, les greffiers et les secrétaires de greffes (p. 35).

58. Voir: Jacques Attali, Une brève histoire de l'avenir, Paris, Fayard, 2009. 
constitue, de ce fait, un déni de justice, de droit et une négation de l'État de droit ${ }^{59}$ et donc de l'État lui-même ${ }^{60}$.

Cette négation quotidienne de l'État de droit dans tous ses aspects soulève la question de l'importance et de l'utilité de la Constitution. Le faible respect de la légalité et la difficile garantie des droits des citoyens par la justice témoignent en effet de l'incapacité de la Constitution à limiter le pouvoir en l'organisant.

\section{L'anéantissement des principes de limitation des pouvoirs}

La limitation du pouvoir politique par la Constitution sous-entend l'encadrement du pouvoir politique par le droit. La Constitution togolaise contient dans ce sens deux principes fondamentaux: la division du pouvoir politique et l'organisation des rapports entre les pouvoirs issus de la division, d'une part, et l'accession au pouvoir par les élections, d'autre part. Malgré l'existence de ces principes, on constate une fusion de fait des pouvoirs et une accession au pouvoir ne correspondant pas au modèle des élections, ce qui conduit à parler de l'artificialité de l'encadrement juridique du pouvoir.

\subsection{L'encadrement juridique du pouvoir politique}

L'encadrement juridique du pouvoir consiste en la division du pouvoir politique en trois pouvoirs et en l'organisation des rapports entre ces différents pouvoirs, d'une part, et en l'établissement des modalités d'accession au pouvoir, d'autre part.

59. Voir Mailis Reps, La Corruption: une menace pour la prééminence du droit, (version provisoire), Commission des questions juridiques et des droits de l'homme, Assemblée parlementaire, Conseil de l'Europe: http://www.assembly. coe.int/CommitteeDocs/2013/NYFf $,\left|\% C_{4}\right|, \mid \% B 1$ nalpressfjdoc1F.pdf (consulté le 24 novembre 2014).

60. Comme le relève le Conseil constitutionnel français dans sa décision du 16 janvier 1982 sur les nationalisations, la protection du droit de propriété constitue l'un des buts de la société politique. Le doyen Favoreu déduit de cette affirmation, le caractère essentiel du droit de propriété parmi les droits et libertés fondamentaux de la société et de l'État. Voir Louis Favoreu, op. cit., p. 407. 


\subsubsection{La division du pouvoir}

La Constitution togolaise du 14 octobre 1992 divise le pouvoir politique en trois et le confie à trois organes différents: le pouvoir législatif au Parlement ${ }^{61}$, le pouvoir exécutif au président de la République et au gouvernement ${ }^{62}$ et le pouvoir judiciaire aux tribunaux ${ }^{63}$. Cette division correspondant à celle opérée par Montesquieu en vue de préserver la liberté politique ${ }^{64}$, un recours à la pensée du baron de La Brède peut faciliter la compréhension de la Constitution togolaise sur ce point.

Une tradition bien établie a déduit du livre XI de L'esprit des lois la théorie de la séparation des pouvoirs dont la paternité est naturellement attribuée à Montesquieu. Cette tradition fait reposer cette théorie sur deux principes permettant la garantie des libertés politiques : l'indépendance des organes et la spécialité des fonctions. Le premier principe exige que chaque organe soit à l'abri des autres et le second que chaque organe exerce exclusivement une fonction, ce qui suppose autant d'organes que de fonctions.

Deux arguments, l'un théorique et l'autre empirique, interdisent le recours à cette interprétation traditionnelle du livre XI de L'esprit des lois pour expliquer le sens de la division du pouvoir politique. Le premier, comme le relève Carré de Malberg, est relatif à l'impossibilité même de l'équilibre car des organes spécialisés ne peuvent se faire équilibre que si les fonctions qu'ils exercent sont équivalentes. Or, comme le note cet auteur, ces fonctions sont hiérarchisées, ce qui implique la hiérarchisation des organes les exerçant. Jean Bodin déduisait cette hiérarchisation des pouvoirs au sein de l'État de la suprématie du pouvoir législatif sur tous les autres pouvoirs.

La Constitution togolaise prévoit, par ailleurs, un certain nombre de mécanismes ne s'accordant pas du tout à ces deux principes. Ces mécanismes sont le pouvoir de dissolution du Parlement par le président de la République (art. 68), la responsabilité du gouvernement devant le Parlement (art. 67), le partage de l'initiative des lois entre le gouvernement et le Parlement (art. 83), le droit d'amendement dont disposent le gouvernement et le Parlement (art. 90), le droit d'accès du gouvernement à l'Assemblée nationale et au Sénat (art. 96). Ces mécanismes impliquent une collégialité (entre les organes exerçant les trois fonctions) incompatible avec les principes d'indépendance des organes et de spécialité des

61. Voir le titre III et principalement l'article 41.

62. Voir le titre IV.

63. Voir le titre VIII.

64. Montesquieu, De l'esprit des lois, livre XI. 
fonctions, d'une part, et concevable avec la thèse de Charles Eisenmann sur la question, d'autre part.

Cet auteur fait reposer la division tripartite du pouvoir politique sur deux principes: la séparation des pouvoirs et la balance des pouvoirs. Il qualifie la séparation des pouvoirs de principe négatif se bornant à poser un interdit: la confusion des pouvoirs. Il qualifie la balance des pouvoirs de principe automatique permettant au pouvoir d'arrêter le pouvoir. Des pouvoirs ne peuvent s'arrêter que s'ils sont distincts et chacun partiellement exercé par chaque organe. Ce qui est recherché ici, ce n'est pas le monopole d'un organe sur un pouvoir ou l'exclusivité de l'exercice, mais bien l'équilibre entre les organes, source de l'équilibre de la société sans laquelle la paix et la liberté politique ne sont pas possibles.

L'explication de la division tripartite du pouvoir politique par les principes de la séparation et de la balance des pouvoirs se comprend aisément lorsqu'on se réfère à l'histoire constitutionnelle et institutionnelle du Togo. La confusion des pouvoirs créée par la Constitution de 1980 est à l'origine des nombreuses atteintes aux droits et libertés qu'il fallait éviter, à tout prix, en séparant les pouvoirs. Mais afin d'éviter que cette séparation ne soit source de dysfonctionnements de l'État, le constituant a pris le soin de confier une parcelle de chaque pouvoir à chacun des organes. L'objectif est de les contraindre à négocier. Il faut en cela voir la volonté du constituant de créer une démocratie de consensus.

\subsubsection{L'établissement des conditions d'exercice du pouvoir politique}

La Constitution indique les modalités de choix des personnes devant exercer chacun des pouvoirs et de règlement des conflits pouvant éventuellement survenir lors de ce choix. L'accession au pouvoir, non par la force, mais selon des procédures préalablement définies par la Constitution constitue la véritable marque de l'institutionnalisation du pouvoir politique et le véritable moyen de préserver la liberté politique des citoyens dont la seule volonté constitue le fondement de l'autorité des pouvoirs publics.

Ces modalités sont décrites par l'article 52 de la Constitution, en ce qui concerne le choix des personnes devant exercer le pouvoir législatif, et les articles 59 à 63 s'agissant du choix du président de la République. Ces dispositions instituent des élections comme modalités de choix et décrivent les conditions d'éligibilité.

L'élection est "un mode de désignation du pouvoir supposant un choix (donc plusieurs possibles) opéré au sein d'un groupe (donc 
un corps électoral) au moyen de mécanismes appropriés permettant d'assurer la mise du vote ${ }^{65}$ ".

Définies comme telles, les élections permettent à la fois le choix des gouvernants et la mise en œuvre des droits du citoyen. Elles se font, donc, selon un modèle citoyen électeur reposant lui-même sur la notion de participation politique. Il faut, dans ce sens, considérer que le citoyen est issu du corps social, il est un membre de la société à la vie de laquelle il a le droit de participer comme l'indiquent les deux premiers paragraphe de l'article 21 de la Déclaration universelle des droits de l'homme: "Toute personne a le droit de prendre part à la direction des affaires publiques de son pays, soit par l'intermédiaire de représentants librement choisis. Toute personne a droit à accéder, dans des conditions d'égalité, aux fonctions de son pays ${ }^{66}$. $\gg$ Ces dispositions laissent entrevoir l'égalité entre les citoyens et plus généralement les droits de l'homme comme fondement du modèle citoyen électeur. Le respect de ces droits se manifeste à travers l'application du principe « un homme, une voix » dans le choix des gouvernants.

L'égalité de droits n'occulte pas la réalité de l'inégalité de talents impliquant chez les citoyens une inégalité dans les aptitudes à exercer certaines fonctions comme l'explique Schumpeter : « La masse du peuple n'élabore jamais sa propre initiative des opinions tranchées [et] elle est encore moins capable de les énoncer, ni de les convertir en attitudes et en actions cohérentes. » Le rôle de cette masse n'est pas de gouverner, mais de produire un gouvernement ${ }^{67}$.

L'article 104 de la Constitution prévoit un règlement juridictionnel de la régularité des élections qu'il confie à la Cour constitutionnelle. Ce contentieux matérialise, in fine, le rejet de la violence comme mode de dévolution du pouvoir ${ }^{68}$ et garantit l'égalité entre les électeurs, l'égalité entre les candidats et la liberté de vote.

Les principes et mécanismes prévus par la Constitution tendent globalement à éliminer la violence dans la société. Du fait de la tradition

65. Laurent Fonbaustier, "Élection», dans Denis Alland et Stéphane Rials (dir.), Dictionnaire de la culture juridique, op. cit., p. 606.

66. Des dispositions similaires se retrouvent dans l'art. 13 de la Charte africaine des droits de l'homme.

67. Voir: Joseph Schumpeter, Capitalisme, socialisme et démocratie [1942], Gaël Fain (trad.), Paris, Payot, 1983.

68. Babacar Gueye, «La démocratie en Afrique : succès et résistances », Pouvoirs, n ${ }^{\circ}$ 129, 2009, p. 12. 
(africaine) entendue, ici, comme un processus de réinvention ${ }^{69}$ et des acculturations modernes, fruit des rencontres entre l'idéologie moderne et des traits non modernes ${ }^{70}$, l'application de ces principes se révèle difficile. Le degré d'acculturation de la conception classique de la Constitution et sa totale réinvention dans le cadre de la tradition les anéantissent.

\subsection{L'artificialité de l'encadrement du pouvoir politique}

L'artificialité de la limitation du pouvoir politique exprime à la fois l'apparent équilibre entre l'Exécutif et le Parlement, d'une part et la désubstantialisation des modalités de dévolution du pouvoir du fait des réalités et contraintes sociales, d'autre part.

\subsubsection{La fusion de fait des pouvoirs}

La balance entre les pouvoirs recherchée par le constituant togolais peine à se matérialiser. Ce retard s'explique par plusieurs raisons. Il faut évoquer, s'agissant de la séparation des pouvoirs, le mouvement général du déclin des parlements prenant plus, au Togo, la forme d'une «appropriation » du Parlement par l'Exécutif ou d'une renonciation du Parlement à ses fonctions ${ }^{71}$. La rareté des lois d'origine parlementaire, des séances de questions au gouvernement, de commissions d'enquêtes parlementaires et l'inactivité des commissions parlementaires témoignent de cette renonciation s'expliquant elle-même par deux raisons. La première est l'incompétence des élus sur les questions souvent débattues à la représentation nationale. Bien des représentants ne disposent pas, en effet, de l'expertise nécessaire à l'exercice de la fonction normative du Parlement et de contrôle de l'action du gouvernement. S'ajoute à cela l'inexpérience de bien des élus dans la fonction de parlementaire. Une précision doit, dans ce sens, être faite. L'expérience parlementaire togolaise ne connaît pas la pratique de jeunes attachés parlementaires apprenant la politique et la fonction de parlementaire à l'ombre et aux cabinets de grands maîtres avant d'exercer, à leur tour, les mêmes fonctions. S'ajoute le fait partisan, amplifié par la concordance continue

69. Jean-François Bayart, «La démocratie à l'épreuve de la tradition en Afrique subsaharienne», Pouvoirs, $\mathrm{n}^{0}$ 129, 2009, p. 128.

70. Voir: Philippe de Lara, "Anthropologie du totalitarisme", Annales. Histoire, sciences sociales, $\mathrm{n}^{\mathrm{o}} 2$, 2008, p. 353-375.

71. Il faut relever que l'autolimitation ne s'est estompée qu'entre 1994 et 2000, période marquée la présence équilibrée d'au moins trois partis politique au Parlement. 
des majorités parlementaire et présidentielle et par une culture locale caractérisée par la double logique selon laquelle, non seulement, il n'est pas bien de contester le chef ${ }^{72}$ mais aussi que «le pouvoir se mange en entier ${ }^{73}$ ». Découle de ces logiques une volonté de l'Exécutif de soumettre l'ensemble des institutions de l'État se matérialisant par une inféodation des pouvoirs législatif et judiciaire.

Cette situation rend impossible la balance des pouvoirs dont la réalisation dépend, en outre, d'autres facteurs comme l'opposition des intérêts économiques et sociaux représentés dans les différents organes ${ }^{74}$, l'équilibre de l'information dont dispose chaque organe et l'impartialité de l'organe chargé de la régulation du fonctionnement des pouvoirs publics institués.

L'opposition des intérêts économiques et sociaux représentés dans les différents organes peut à la fois renvoyer à l'équilibre du partage des compétences financières entre l'Exécutif et le Législatif et à l'équilibre des moyens financiers entre la majorité présidentielle et l'opposition. Le déséquilibre du partage des compétences financières entre l'Exécutif et le Parlement étant avéré ${ }^{75}$, il ne reste qu'à rechercher l'équilibre des pouvoirs financiers entre la majorité présidentielle et son opposition. Des différentes forces politiques représentées dans les institutions de l'État, la majorité présidentielle dispose, on le suppose, de plus de moyens financiers. Cette supposition se fonde à la fois sur le poids de l'appareil d'État dans l'économie et sur la pratique intensive de l'accumulation économique constatée, par Jean-François Bayard ${ }^{76}$, chez

72. Palouki Massina évoque dans ce sens «la crainte révérencieuse que les populations éprouvent à l'égard de la puissance publique» et la généralité du «sentiment [...] selon lequel l'action du détenteur de l'autorité ne saurait être critiquée». Voir: Palouki Massina, op. cit., p. 17.

73. Voir sur ce point, voir: Vincent Foucher, «Difficiles successions en Afrique subsaharienne. Persistance et reconstruction du pouvoir personnel ", Pouvoirs, $\mathrm{n}^{\mathrm{o}} 129,2009, \mathrm{p} .129$. L'auteur constate le règne de cette logique, à quelques exceptions près, dans l'ensemble des pays d'Afrique subsaharienne. Il indique dans ce sens que dans ces pays, «la casquette du Prince et celle de l'État ne sont guère séparées" (p. 30).

74. Michel Troper, «Séparation des pouvoirs", dans Dictionnaire électronique Montesquieu, Catherine Volpilhac-Auger (dir.), ENS de Lyon, septembre 2013: http:// dictionnaire-montesquieu.ens-lyon.fr/fr/article/1376427308/fr/ (consulté le 24 novembre 2014).

75. Sur ce point, voir: Sasso Pagnou, La Gestion des finances publiques au Togo: un système à rénover, Éditions universitaires européennes, 2011, p. 132 et suiv.

76. Jean-François Bayard, «Les sociétés africaines face à l'État », Pouvoirs, $\mathrm{n}^{\circ} 25,1983$, p. 25. Voir également du même auteur, L'État en Afrique. La politique du ventre, Paris, Fayard, 2006, p. 99 et suiv. 
les détenteurs du pouvoir dans les pays d'Afrique subsaharienne ${ }^{77}$. Le déséquilibre, en faveur de l'Exécutif dans le partage des compétences financières et le poids de l'appareil d'État dans l'économie créent une asymétrie de l'information financière ${ }^{78}$.

Les liens entre le pouvoir politique et le pouvoir financier ${ }^{79}$, ou mieux la dépendance du premier du second, conduisent à affirmer la suprématie de l'Exécutif. La réalisation de la balance des pouvoirs dépendra alors de l'impartialité de l'organe régulateur du fonctionnement des pouvoirs publics institués.

L'affirmation de l'impartialité de l'organe régulateur du fonctionnement des pouvoirs publics dépend de l'idée que l'on se fait de la façon de trancher du juge. Est-il lié au droit ou prend-il ses décisions pour des raisons de pure opportunité?

Selon la théorie réaliste de l'interprétation ${ }^{80}$, les décisions du juge ont toujours des enjeux sociaux. Il ne peut donc être neutre. Cette position est bien résumée par Gwénaëlle Calves: «La Cour ne dit pas le droit dans une salle de classe. Elle est prise dans un jeu de forces qui lui impose, bien souvent, d'avancer masquée ou, à tout le moins, de développer une stratégie jurisprudentielle ${ }^{81}$.» Le doyen Favoreu relève aussi une part d'opportunité dans les décisions du juge constitutionnel en se fondant sur la notion d' "erreur manifeste " à laquelle le conseil a eu à maintes reprises à se référer et qui «se trouve à la frontière de

77. S’agissant spécialement du Togo, le phénomène est décrit, notamment, par Gilles Labarthe. L'auteur évoque (en se référant à certains témoignages) une fortune présidentielle faramineuse construite sur l'argent des phosphates frauduleusement viré sur des comptes personnels en Suisse. Voir: Gilles Labarthe, Le Togo, de l'esclavage au libéralisme mafieux, Marseille, Agone, 2005, p. 99 et suiv.

78. Sur ce point, voir Sasso Pagnou, op. cit., p. 173.

79. Sur ce point, voir: Michel Bouvier et Robert Hertsog, «Réforme des finances publiques: réforme de l'État», RF fin. publ. 2001, p. 3. Voir également Michel Bouvier, "Mutations des finances publiques et crise du pouvoir politique? », RF fin. publ. 2002, p. 241.

80. La théorie réaliste de l'interprétation du juge, développée aux États-Unis, accorde au juge une grande liberté d'interprétation des textes. Elle rassemble de ce fait une thèse négative réfutant le formalisme et le droit naturel et une thèse positive selon laquelle le droit est produit par les juges. Aucun sens, aucune signification ne s'impose d'elle-même au juge interprète selon les réalistes. John Chipman Gray écrit dans ce sens: "les lois énoncent des mots et il revient au juge de dire ce que les mots signifient» (cité par Pierre Brunet, «La théorie réaliste n'est-elle qu'une théorie de l'interprétation? », dans Jean-Jacques Sueur et Pascal Richard (dir.), La transgression, Bruxelles, Larcier, 2013, p. 397-414). L'avantage de cette théorie est la connaissance objective du droit qu'elle permet.

81. Voir : Gwénaële Calves, «Le juge constitutionnel entre droit et politique : une brève présentation de la querelle des principes neutres", Jus politicum, $\mathrm{n}^{\circ}{ }_{5}$, 2010, p. 28. 
la légalité et de l'opportunitéé ${ }^{82}$. Cette part d'opportunité peut être un frein à la neutralité et à l'impartialité du juge et l'empêcher, de ce fait, de garantir la balance des pouvoirs publics dont la régulation $\mathrm{du}$ fonctionnement lui incombe. Dans les faits, le juge constitutionnel togolais conscient du déséquilibre des forces sur le terrain et, surtout, instruit des épisodes du 3 décembre $1991^{83}$, du 22 et 23 octobre $1992^{84}$ et du 5 février $2005^{85}$ est contraint d'être réaliste dans ses décisions ${ }^{86}$. Ces événements renvoient à l'armée dont les différentes interventions dans l'histoire politico-institutionnelle du Togo invitent à repenser la théorie de la séparation des pouvoirs au Togo et de façon plus large dans bien de pays africains.

\subsubsection{Le dévoiement des modalités d'exercice du pouvoir}

Aux termes des articles 52 et 59 de la Constitution, le président de la République et les députés sont élus au suffrage universel direct et secret. Cette exigence permet le respect de l'égalité et de la liberté de vote considérés comme des principes fondamentaux du droit électoral ${ }^{87}$ et donc, la substance même des élections.

En matière électorale, le principe d'égalité est souvent évoqué au sujet du décompte des voix et du taux de représentativité des élus. Il convient d'ajouter, aujourd'hui, à ces deux éléments les moyens

82. Louis Favoreu, op. cit., p. 396.

83. Voir: Cornélius Azaglo, "Chronique d’un putsch annoncé », Togo-Presse, n 3557 du 9 décembre 1992.

84. Le Haut-Conseil de la République (Assemblée nationale de transition) est pris en otage par les militaires. Voir: Maurice Engueleguele et alii, «République du Togo. Bilans annuels de 1983 à 2013 ", L'État du monde, Paris, La Découverte, 2013.

85. «Déclaration des forces armées togolaises (FAT). Le choix de des forces armées togolaises porté sur Faure Gnassingbé », Togo-Presse, nº 6994 du lundi 9 décembre 1991.

86. On constate le même réalisme chez le juge constitutionnel ivoirien pendant la crise post-électorale de 2011. Après avoir reconnu la victoire de Laurent Gbagbo, le 3 décembre 2010, il reconnaît, quelques mois plus tard, celle d'Alassane Ouattara après sa victoire militaire. Il explique la reconnaissance d'Alassane Ouattara par le fait que ce dernier est reconnu par l'ensemble de la communauté internationale. Les décisions « contraignantes" de l'Union africaine, avancées par Ndré pour expliquer ce revirement rendent compte des pressions pouvant orienter la décision du juge constitutionnel dans certains pays africains.

87. Voir: Richard Ghevontian, "La notion de sincérité du scrutin ", Cah. Cons. const. 2003, $\mathrm{n}^{\mathrm{o}} 13$, p. 1: http://www.conseil-constitutionnel.fr/ conseil-constitutionnel/francais/nouveaux-cahiers-du-conseil/cahier-n-13/ la-sincerite-du-scrutin-etudes-reunies-et-presentees-par-richard-ghevontian. 52034.html (consulté le 24 novembre 2014). 
financiers nécessaires à une communication déterminant de plus en plus le choix des électeurs ${ }^{88}$ dans la mesure où tant l'article 2 de la Constitution que l'article 1 de la Déclaration universelle des droits de l'homme de 1948 interdisent que les déséquilibres sociaux, ici celui des moyens financiers, introduisent une inégalité dans la jouissance des droits.

Le décompte de voix renvoie aux modes de scrutin permettant le passage du décompte des voix à la désignation des élus. L'égalité voudrait, ici, que tous les électeurs disposent du même poids. Dans le cadre des élections législatives où est pratiquée la proportionnelle à la plus forte moyenne, cette égalité est difficile à atteindre du fait de sa tendance à favoriser les grands partis comme en témoigne souvent la disproportion entre le nombre de voix obtenues et celui des sièges attribués.

Tableau 1 - Nombre de voix et sièges attribués par parti.

\begin{tabular}{lccccc}
\hline Partis & Unir & CST & Arc-en-ciel & UFC & Sursaut \\
\hline Voix & 850000 & 800000 & 200000 & 149256 & 14420 \\
\hline Sièges & 62 & 19 & 6 & 3 & 1 \\
\hline
\end{tabular}

La forte disparité entre les ratios du nombre de voix obtenues par chaque parti sur le nombre de sièges qui lui sont attribués (voir le tableau 1) rompt l'égalité entre les électeurs et les partis politiques. La petitesse des circonscriptions électorales participe à ce phénomène dans la mesure où elle conduit à beaucoup d'annulation de vote. Un autre découpage avec la région ou le territoire national comme circonscription électorale aurait permis une avancée vers l'égalité. L'atteinte de l'égalité est dans ce sens conditionnée par le choix du mode de scrutin. Mais le véritable problème que soulève ce mode de scrutin dans le contexte togolais n'est pas tant les inégalités qu'il entraîne que l'impact des connaissances et de la culture des acteurs politiques sur la mise en œuvre du principe d'égalité en matière électorale. Ce mode de scrutin était exigé, par l'opposition aux accords de Ouagadougou, du fait de

88. André Gosselin, «La publicité électorale», Les études de la communication publique, cahier $\mathrm{n}^{0} 11,1997$, p. 1-24: http://www.com.ulaval.ca/fileadmin/contenu/docs_ pdf/articles/etudes_com_publ/11ecp.pdf (consulté le 24 novembre 2014). Voir également Antoine Socpa, "Les dons dans le jeu électoral au Cameroun", Cahiers d'études africaines, $\mathrm{n}^{\circ} 157,2000$, p. 91-108. L'auteur relève un lien entre les dons et les consignes de votes, constatable aussi au Togo: «Mangez, buvez, et votez pour mon parti!» (p. 97). 
sa capacité à permettre le respect du principe d'égalité. L'inculture du personnel politique peut donc constituer un frein à la mise en œuvre des principes constitutionnels.

L'égalité dans le décompte doit être précédée de l'égalité de représentation, c'est-à-dire de l'équité des différences de représentativité (ratios nombre d'habitants/élus) d'une circonscription électorale à l'autre. Le respect de cette égalité - droit civil et politique ${ }^{89}$ - passe par la conformité du découpage électoral au principe d'équilibre démographique ${ }^{90}$.

Tableau 2 - Représentativité des différentes circonscriptions ${ }^{91}$.

\begin{tabular}{lrrr}
\hline Circonscriptions & Habitants & Sièges & Représentativité \\
\hline Grand Lomé & 1570285 & 10 & 157028,50 \\
\hline Tône-Cink & 364000 & 4 & 91000,00 \\
\hline Yoto & 165596 & 3 & 88532,00 \\
\hline Ogou Anié & 321308 & 4 & 80000,00 \\
\hline Kozah & 225000 & 3 & 75000,00 \\
\hline Zio & 295177 & 4 & 73794,25 \\
\hline Tchaoudjo & 190114 & 3 & 63371,30 \\
\hline Tandjaoré & 117000 & 2 & 58500,00 \\
\hline Blitta & 137000 & 3 & 45000,60 \\
\hline Assoli & 51000 & 2 & 25500,00 \\
\hline Danyi & 38742 & 2 & 19371,00 \\
\hline
\end{tabular}

Les chiffres du tableau 2 indiquent de grandes disparités de représentativité d'une circonscription à l'autre. La représentativité dans le

89. Voir Cons. const., déc. 1-2 juillet 1986, $\mathrm{n}^{0}$ 86-208 DC et déc. 18 novembre 1986, $\mathrm{n}^{\circ} 86-218$ DC.

90. Se fondant sur l'article $1^{\text {er }}$ de la Déclaration des droits de l'homme et du citoyen et les articles 1 et 3 de la Constitution française (dispositions aussi présentes dans la Constitution togolaise), le Conseil constitutionnel français a érigé l'égalité devant le suffrage en exigence constitutionnelle, de laquelle il fait découler un principe d'équilibre démographique. Il affirme, dans ce sens, que l'Assemblée nationale doit "être élue sur des bases essentiellement démographiques; que, si le législateur [pouvait] tenir compte d'impératifs d'intérêt général susceptibles d'atténuer la portée de cette règle fondamentale, il ne saurait le faire que dans une mesure limitée et en fonction d'impératifs précis » (Cons. const., déc. 18 novembre 1986, $\mathrm{n}^{\text {os }} 86-208$ DC et 86-218 DC).

91. Les chiffres indiqués dans ce tableau sont issus des résultats définitifs du recensement général de la population et de l'habitat du 6 au 21 novembre 2010 et du décret $\mathrm{n}^{\mathrm{o}}$ 2013020/PR portant répartition des sièges de députés à l'Assemblée nationale par circonscription électorale. Figurent dans ce tableau, en plus du Grand Lomé, la plus grande et la plus petite circonscription électorale de chacune des cinq régions. 
Grand Lomé est le double de la moyenne nationale de représentativité, 60 034, obtenue en divisant le nombre de la population togolaise par le nombre de députés à l'Assemblée nationale (soit 6191155 par 91). On constate parallèlement que la représentativité de l'Assoli fait le tiers de la représentativité moyenne nationale et le sixième de la représentativité du Grand Lomé. Le principe d'égalité semble donc peu respecté.

L'une des raisons de ces disparités est le calquage du découpage électoral sur le découpage des circonscriptions administratives correspondant lui-même plus ou moins à un découpage ethnique. Une égalité de représentativité pourrait conduire à des disparités de représentation ethnique, ce qui ne manquerait pas d'être instrumentalisée ${ }^{92}$ et de mettre en péril l'unité nationale ${ }^{93}$. L'unité nationale constituerait, de ce fait, un impératif d'intérêt général permettant d'atténuer la règle fondamentale de l'élection de l'Assemblée nationale sur des bases essentiellement démographiques ${ }^{94}$ si les écarts n'étaient pas exorbitants ${ }^{95}$.

On ne saurait passer sous silence l'ineffectivité (voulue ?) de la limitation des dépenses de campagnes électorales prévues par l'article 120 de la loi $\mathrm{n}^{0}$ 2012-002 portant Code électoral sous le contrôle du juge financier ${ }^{96}$. Ces limitations tendent, comme précédemment indiqué, à assurer une égalité entre les candidats ou du moins à éviter que des inégalités sociales ne créent des inégalités dans la jouissance des droits civils et politiques. L'absence de contrôle des dépenses de campagnes par la Cour des comptes ${ }^{97}$ peut conduire au dépassement des limites fixées par le Code électoral et surtout à un déséquilibre de moyens

92. Comme le démontre G. Labarthe, les politiques togolais ont, depuis la lutte pour les indépendances, récupéré la vieille technique du colon français, «diviser pour régner» en instrumentalisant les ethnies. Voir Gilles Labarthe, op. cit., p. 54.

93. Sur les liens entre l'instrumentalisation des ethnies et la mise en péril de l'unité nationale, se référer notamment à Jean-Loup Amselle et Elikia M’Bokolo (dir.), Au cour de l'ethnie: ethnies, tribalisme et État en Afrique, Paris, La DécouverteMaspero, 1985.

94. Voir Cons. const., déc. 2 juillet 1986, $\mathrm{n}^{\circ}$ 86-208 DC.

95. En France, l'écart autorisé d'une circonscription à l'autre est de $20 \%$ de la population moyenne du département. Aucune limitation de ce genre n'existe dans les textes togolais. Mais on peut raisonnablement estimer exorbitant le fait qu'une circonscription électorale soit le double, c'est-à-dire $100 \%$ de la moyenne nationale.

96. Art. 120 et 122.

97. Celle-ci n'a publié aucun rapport sur les comptes de campagnes des candidats et des partis. Cela peut s'expliquer par l'absence d'établissement de comptes de campagne par les partis politiques ou par l'impotence des organes de contrôles sur les finances publiques. Sur ce dernier point, voir Sasso Pagnou, "Les récentes évolutions de la gestion publique financière au Togo", Revue électronique Afrilex, 2012, $2^{\mathrm{e}}$ numéro spécial sur les finances publiques: 
financiers entre les différents adversaires. Il est possible, en se référant aux liens intrinsèques entre l'exercice du pouvoir politique et l'accumulation des richesses relevés par Bayard dans les pays de l'Afrique subsaharienne, d'émettre l'hypothèse de la participation des deniers publics à ce déséquilibre. L'hypothèse semble confirmée dans les faits par la disproportion des moyens financiers entre la majorité au pouvoir et son opposition. Cette violation indirecte de l'article $72 \mathrm{du}$ Code électoral, interdisant l'utilisation des biens ou moyens d'une personne morale de droit public, d'une institution ou d'un organisme aux fins de propagande pour influencer ou tenter d'influencer le vote, entache les élections d'une iniquité contraire à l'exigence d'égalité.

L'exigence de liberté, second principe fondamental du droit électoral, est neutralisée par un certain nombre de réalités. Il faut, pour comprendre cette neutralisation, rappeler la distinction faite par Isaiah Berlin ${ }^{98}$ entre la définition libérale de la liberté dite négative et celle romantique dite positive.

La définition libérale de la liberté met l'accent sur la reconnaissance des droits et libertés à l'individu. La liberté à laquelle renvoie cette définition est dite négative en ce qu'il est interdit à autrui de s'interférer dans les droits et libertés reconnus à cet individu. Quant à la définition romantique, c'est-à-dire la liberté positive, elle met l'accent sur la capacité effective de l'individu de jouir des droits et libertés qui lui sont reconnus.

Le droit et la liberté de vote correspondent à la première définition, c'est-à-dire à la liberté négative. Cette liberté est formelle ${ }^{99}$. Elle se limite, en effet, à la reconnaissance des droits et libertés et laisse de côté les pesanteurs socio-économiques pouvant altérer la capacité d'analyse de l'individu, aliéner sa pensée et l'empêcher de jouir de ses droits et d'exercer ses libertés. La reconnaissance et même la garantie officielle (absence de pression et garantie du secret de vote par les urnes) du droit et de la liberté de vote n'attestent pas, de ce fait, de leur effectivité. La certification de cette effectivité exige la recherche de la liberté réelle. Il est alors nécessaire de recourir à la définition romantique de la liberté dite liberté positive ou encore liberté capacité.

http://afrilex.u-bordeaux4.fr/sites/afrilex/IMG/pdf/5-_Les_recentes_evolutions_ de_la_gestion_publique_financiere_au_Togo.pdf (consulté le 24 novembre 2014).

98. Voir: Isaiah Berlin, Deux concepts de la liberté, texte de la leçon inaugurale prononcée à l'université d'Oxford le 31 octobre 1958.

99. Voir, sur ce point, John Rawls, Théorie de la justice [1971], Catherine Audard (trad.), Paris, Seuil, « Points », 2009. 
La liberté positive repose fondamentalement sur l'«approche des capabilités ». Amartya Sen ${ }^{100}$, l'auteur de cette approche, résume la liberté réelle d'un homme par sa capacité interne d'effectuer un choix autonome entre plusieurs possibilités s'offrant à lui. Cette liberté repose donc non seulement sur la capacité de pouvoir choisir, ce qui suppose l'existence de plusieurs possibilités de choix, mais aussi sur la possibilité de pouvoir effectuer un choix autonome, c'est-à-dire conscient et réfléchi.

À une partie non négligeable de Togolais, surtout à ceux des villes et villages de l'intérieur du pays, seule s'offre une possibilité, celle du parti au pouvoir totalement implanté sur l'ensemble du territoire grâce à ses énormes moyens financiers vraisemblablement attribuables au phénomène d'accumulation sus évoqué. L'omniprésence d'un parti et le manque de visibilité des autres partis conduisent à un système de parti unique de fait qui, en vrai système de pensée unique, n'offre qu'une possibilité dont l'acception est favorisée par la pauvreté, l'illettrisme, le manque d'information et par bien d'autres phénomènes aliénant le citoyen-électeur, viciant son raisonnement et détournant sa liberté de choix. L'effectivité de la liberté du citoyen dépend alors de son cadre social et de son niveau de vie, bref de la satisfaction de ses droits économiques et sociaux. Il est possible, dans ce sens, d'expliquer l'incapacité de la Constitution à garantir la liberté politique par la culture des dirigeants et des citoyens ${ }^{101}$ et la qualité de la vie sociale. Si l'on considère la culture comme ce qui est de l'ordre de l'acquis et non de l'inné, la garantie de la bonne application des valeurs et principes constitutionnels dépend non seulement du niveau d'instruction des acteurs mais de leur éducation dépendant globalement des valeurs familiales qu'ils ont reçues. Le tribalisme, la corruption et la violence ${ }^{102}$ qui rendent difficile la pleine application des principes constitutionnels germent souvent dans la famille. La famille doit donc être au centre du projet de restauration de l'autorité de la Constitution dans la mesure où elle est la «communauté naturelle au sein de laquelle s'expérimente la sociabilité humaine » et peut de ce fait constituer « la meilleure garantie

100. Voir: Amartya Sen, Éthique et économie et autres essais, Paris, PUF, 2012; ou du même auteur, L'Idée de justice, Paris, Flammarion, 2012.

101. Sur l'importance de la formation pour le respect de la Constitution, voir : Adama Kpodar, "Bilan sur un demi-siècle de constitutionnalisme en Afrique noire francophone" art. cité, p. 23. L'auteur cite notamment Slobodan Milacic pour lequel, "au-delà du juridique et du politique même, c'est la culture qui est la meilleure garantie de la bonne application des valeurs et principes».

102. Voir: Comi M. Toulabor, «Violence militaire, démocratisation et ethnicité au Togo », Autrepart, n 10, 1999, p. 105-115. 
contre toute dérive de type individualiste ou collectiviste ${ }^{103}$ ». Cette importance de la famille se retrouve à travers cette affirmation de JeanJacques Rousseau : «La famille est donc, si l'on veut, le premier modèle des sociétés politiques : le chef est à l'image du père, le peuple est l'image des enfants; et tous étant nés égaux et libres, n'aliènent leur liberté que pour leur utilité $\mathrm{104}^{\text {.» }}$

Il faut donc rappeler, au terme de cette analyse, deux réalités dont l'insuffisante prise en compte, dans le contexte togolais, explique la survivance de pratiques contraires à l'esprit et à la lettre de la Constitution du 14 octobre 1992. La première est que l'« on ne change pas une société par décret ${ }^{105}$ ». La tentative de passer directement d'un État de confusion de pouvoirs et d'absolutisme, avec la même classe politique et sans une préparation à la citoyenneté et aux valeurs démocratiques, à une société libérale était vouée d'avance à l'échec. La survivance des pratiques contraires à la Constitution découle de la survivance des anciennes mentalités. La restauration de l'autorité de la Constitution exige, dans ce sens, un changement des mentalités.

La seconde réalité est la réciprocité des influences entre la Constitution et la société. S'il est vrai que la Constitution garantit l'équilibre de la société, l'équilibre social garantit aussi l'équilibre constitutionnel ${ }^{106}$. La balance des pouvoirs étant ineffective du fait des déséquilibres sociaux (déséquilibre des moyens financiers entre les partis politiques et entre les personnes exerçant chacun des pouvoirs, la pauvreté de la population, l'illettrisme d'une bonne partie de la population...), il paraît nécessaire, si l'on veut rétablir l'équilibre constitutionnel de rétablir, préalablement, l'équilibre social. La Constitution ne peut, en effet, acquérir toute son autorité et la démocratie être effective sans une éradication de la pauvreté matérielle et surtout mentale de la population et des élus, tant il est vrai $\mathrm{qu}^{\prime}$ « il faut un minimum de confort pour pratiquer la vertu ${ }^{107} »$.

Enseignant-chercheur Faculté de droit et des sciences politiques Université de Kara (Togo)

103. Voir : Conseil pontifical, «Justice et paix», dans Compendium de la doctrine sociale de l'Église, Paris, Cerf, 2005.

104. Voir Jean-Jacques Rousseau, Du contrat social, livre 1, chap. 1.2.

105. Voir : Michel Crozier, On ne change pas la société par décret, Paris, Grasset, 1979.

106. Voir : Michel Troper, «Séparation des pouvoirs», art. cité.

107. Saint Thomas d'Aquin. 